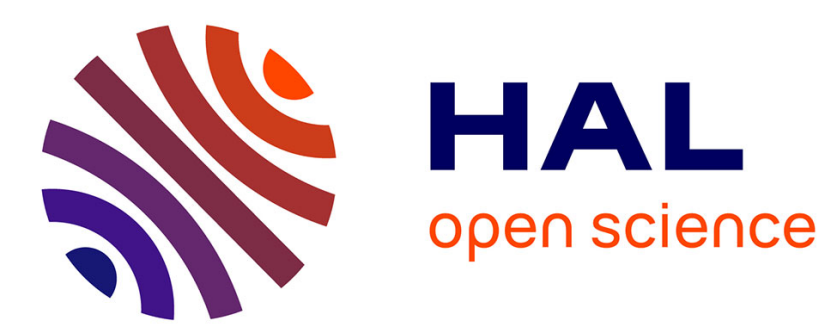

\title{
Optimality of affine control system of several species in competition on a Sequential Batch Reactor
}

\author{
J.C. Rodriguez, Hector Ramirez, Pedro Gajardo, Alain Rapaport
}

\section{To cite this version:}

J.C. Rodriguez, Hector Ramirez, Pedro Gajardo, Alain Rapaport. Optimality of affine control system of several species in competition on a Sequential Batch Reactor. International Journal of Control, 2014, 87 (9), pp.1877-1885. 10.1080/00207179.2014.891360 . hal-01088224

\section{HAL Id: hal-01088224 \\ https://hal.inria.fr/hal-01088224}

Submitted on 27 Nov 2014

HAL is a multi-disciplinary open access archive for the deposit and dissemination of scientific research documents, whether they are published or not. The documents may come from teaching and research institutions in France or abroad, or from public or private research centers.
L'archive ouverte pluridisciplinaire $\mathbf{H A L}$, est destinée au dépôt et à la diffusion de documents scientifiques de niveau recherche, publiés ou non, émanant des établissements d'enseignement et de recherche français ou étrangers, des laboratoires publics ou privés. 


\title{
Optimality of affine control system of several species in competition on a Sequential Batch Reactor
}

\begin{abstract}
In this paper we analyze the optimalty of affine control system of several species in competition for a single substrate on a Sequential Batch Reactors (SBR), with the objective being to reach a given (low) level of the substrate. We allow controls to be bounded measurable functions of time plus possible impulses. A suitable modification of the dynamics leads to a slightly different optimal control problem, without impulsive controls, for which we apply different optimality conditions derived from Pontryagin principle and the Hamilton-Jacobi-Bellman equation. We thus characterize the singular trajectories of our problem as the extremal trajectories keeping the substrate at a constant level. We also establish conditions for which a Immediate One impulse (IOI) strategy is optimal. Some numerical experiences are then included in order to illustrate our study and show that those conditions are also necessary to ensure the optimality of the $I O I$ strategy.
\end{abstract}

Subject classification: 49J15, 49J20, 97M10

Key words: affine control systems, singular trajectories, optimal control problem, sequential batch reactors, pontryagin maximum principle.

\section{Introduction.}

In this paper, we focus on optimal control problem for an affine control system where several species compete for a single substrate on a Sequential batch reactor, the objective being to reach a given (low) level of the substrate.

The Sequential batch reactor (SBR) is a device that consists typically in a tank which is filled with biological micro-organisms capable to degrade some undesirable substrate. The method consists then in a sequence of cycles composed by three phases: - Phase 1: filling the reactor with water to be treated, - Phase 2: waiting the concentration of the undesirable substrate to decrease until a given (low) concentration, - Phase 3: emptying the reactor from the clean water, leaving the sludge inside.

The Sequential batch reactor are often used in biotechnological application, notably in waste-water treatments. See [6], [9], [11], [15] for more details about fundamental role of SBR in bioengineering.

We consider only increasing growth functions for these species. So, this paper extends some results obtained for one and two species in [9] and [11]. For a multi-species setting, we fully characterize the existence of singular arc (which existence can lead to 
complex solutions that are not easily tractable from the mathematical point of view). The extremal trajectories of the singular arc type are characterized as the strategies used to maintain the substrate at a constant level, and we study under which conditions the strategy that consists of filling as fast as possible the tank up to its maximum capacity and then waiting is optimal. This strategy is called on Immediate One Impulsive (IOI) in the impulsional framework developed here and it will be precisely defined in Section 5. The last issue has been sucessfully solved for one single species in [9] by only assuming that the growth function is nondecreasing. However, when two species coexist in the SBR, it is shown in [11] that the fact that both growth functions are increasing is no longer enough to ensure the optimality of the IOI strategy. In Section 5, we have only proved that the IOI strategy is optimal when one species is highly performant and the others all are sufficiently close one to each other, which somehow captures the two species case. However, the study of IOI and singular strategies for our problem in which some of the functions of growth could be Haldane type and all remaining being increasing functions must be analyzed to obtain new optimal strategies in the presence of several species, and this will be done in future research by imposing appropriate additional conditions on growth functions or their derivatives, and the use of higher-order optimality conditions of affine control systems, the envelope theory, symplectic geometric methods. See for instance [2], [5] and references therein.

\section{Formulation of the optimal control problem}

In this section we quickly describe the model and the optimal control problem involved in our analysis.

We consider a SBR with several species in competition for a single substrate. The dynamics of this process can be described as follows (see [15])

$$
\left\{\begin{array}{l}
\dot{x}_{i}=\mu_{i}(s) x_{i}-\frac{F}{v} x_{i}, x_{i}\left(t_{0}\right)=x_{i 0} \quad(i=1 \cdots n), \\
\dot{s}=-\sum_{j=1}^{n} \mu_{j}(s) x_{j}+\frac{F}{v}\left(s_{i n}-s\right), s\left(t_{0}\right)=s_{0} \\
\dot{v}=F, v\left(t_{0}\right)=v_{0}
\end{array}\right.
$$

where $s$ and $v$ stand, respectively, for the concentration of the substrate and the current volume of water present in the tank. The parameter $s_{i n}>0$ is a constant which represents the substrate concentration in the input flow. The concentration of the $i$ th species is denoted by $x_{i}$ whose growth functions $\mu_{i}(\cdot)$ are non-negative smooth functions such that $\mu_{i}(0)=0$, and the input flow $F$ is a non-negative control variable.

Given a (desirable) substrate concentration $\left.s_{\text {out }} \in\right] 0, s_{i n}[$ and a volume (of the reactor) $v_{\text {max }}>0$, consider the domain $\left.\left.\left.\mathcal{D}=\left(\mathbb{R}_{+}^{n} \backslash\{0\}\right) \times\right] 0, s_{i n}\right] \times\right] 0, v_{\text {max }}[$ and the target $\mathcal{T}=$ $\left.\left.\mathbb{R}_{+}^{n} \times\right] 0, s_{\text {out }}\right] \times\left\{v_{\max }\right\}$. From any initial condition $\xi=\left(x_{10}, \ldots, x_{n 0}, s_{0}, v_{0}\right)$ in $\mathcal{D}$ at time $t_{0}$, the objective is to reach $\mathcal{T}$ in minimal time. This means to treat as fast as possible the maximum quantity of water, which in the case of the considered SBR is given by $v_{\max }$. 
In the context of optimal control theory it leads to the following optimization problem

$$
\inf _{F(\cdot)}\left\{t-t_{0} \mid s^{t_{0}, \xi, F}(t) \leq s_{\text {out }}, v^{t_{0}, \xi, F}(t)=v_{\max }\right\},
$$

where $s^{t_{0}, \xi, F}(\cdot), v^{t_{0}, \xi, F}(\cdot)$ denote solutions of $(2.1)$ with initial condition $\xi \in \mathcal{D}$ at time $t_{0}$ and control $F(\cdot)$.

Here $F(\cdot)$ is allowed to be a non-negative measurable function plus possible positive impulses. Indeed, instead of an ordinary control $F(\cdot)$, we consider a measure $d F(\cdot)$ that we decompose into a sum of a measure absolutely continuous with respect to the Lebesgue measure $u(t) d t$ and a singular or impulsive part $d \sigma$ (see $[12,13])$

$$
d F(t)=u(t) d t+d \sigma,
$$

where, $u(\cdot)$ is a measurable non-negative control that we impose to be bounded from above by $u_{\max }$, because it corresponds to the use of a pump device. At time $t$, the nonnegative impulse $d \sigma$ corresponds to an (instantaneous) addition of volume from $v^{-}(t)$ to $v^{+}(t)$.

From [11] we know that a time parameterization $\tau \geq t_{0}$ such that $d t=r(\tau) d \tau$ with

$$
r(\tau)= \begin{cases}1 & \text { when the pump device is used } \\ 0 & \text { when an impulse is used }\end{cases}
$$

permits to replace dynamics (2.1) by the system

$$
\left\{\begin{array}{l}
\frac{d x_{i}}{d \tau}=r \mu_{i}(s) x_{i}-\frac{u}{v} x_{i} \quad(i=1 \cdots n), \\
\frac{d s}{d \tau}=-r \sum_{j=1}^{n} \mu_{j}(s) x_{j}+\frac{u}{v}\left(s_{i n}-s\right), \\
\frac{d v}{d \tau}=u,
\end{array}\right.
$$

where the controls $u(\cdot)$ and $r(\cdot)$ are sought among measurable functions w.r.t. $\tau$, taking values in $\left[0, u_{\max }\right]$ and $\{0,1\}$, respectively. Notice that in this formulation $u(\cdot)$ plays the both role of an ordinary control when $r=1$ and the control of the amplitude of the jump when $r=0$, with the same single constraint $u \in\left[0, u_{\max }\right]$.

Next, the system (2.2) can be rewritten as the following affine control system

$$
\dot{\mathbf{z}}=u_{1} G_{1}(\mathbf{z})+u_{2} G_{2}(\mathbf{z})
$$

where $u_{1}=r, u_{2}=u, \mathbf{z}^{\top}=\left(x_{1}, \cdots, x_{n}, s, v\right)$, and $G_{1}(\mathbf{z}(\tau)), G_{2}(\mathbf{z}(\tau))$ be the vector fields defined, respectively, by 


$$
G_{1}(\mathbf{z}(\tau))=\left(\begin{array}{c}
\mu_{1}(s) x_{1} \\
\vdots \\
\mu_{n}(s) x_{n} \\
-\left(\sum_{i=1}^{n} \mu_{i}(s) x_{i}\right) \\
0
\end{array}\right), \quad G_{2}(\mathbf{z}(\tau))=\left(\begin{array}{c}
-\frac{x_{1}}{v} \\
\vdots \\
-\frac{x_{n}}{v} \\
\frac{\left(s_{i n}-s\right)}{v} \\
1
\end{array}\right)
$$

The formalism given by system (2.3) will be intensively exploited in the next sections.

Remark 1 Any trajectory of (2.3) with initial condition $\xi=\left(x_{10}, \ldots, x_{n 0}, s_{0}, v_{0}\right) \in \mathcal{D}$ lies in the region defined by:

$$
\rho(\xi)=v\left(\sum_{j=1}^{n} x_{j}+s-s_{i n}\right)=w\left(\sum_{j=1}^{n} y_{j}+z-s_{i n}\right) .
$$

This leads to a reduction of variables in dynamics (2.2), which will be used in Section 5.

Remark 2 Since one can always take $r=0$ and $u=0$ on a arbitrarily large $\tau$-interval without modifying the total time, the minimal time problem has no unique solution. Hence, we will be only interested in controls satisfying $r(\tau) \neq 0$ or $u(\tau) \neq 0$ for all time $\tau$.

Consequently, from now on, we work with $V(\cdot)$ the value function of the reformulated problem (2.3) given by

$$
V(\xi)=\inf _{(u, r)(\cdot)}\left\{\int_{t_{0}}^{\tau} r(\theta) d \theta \mid s^{t_{0}, \xi, u, r}(\tau) \leq s_{\text {out }}, v^{t_{0}, \xi, u, r}(\tau)=v_{\max }\right\},
$$

where $s^{t_{0}, \xi, u, r}(\cdot), v^{t_{0}, \xi, u, r}(\cdot)$ denote solutions of (2.3) with initial condition $\xi \in \mathcal{D}$ at time $t_{0}$ and controls $u(\cdot)$ and $r(\cdot)$.

\section{Generalities and Pontryagin's Maximum Principle}

In this section we recall the main notions and some results in [11] in order to have a self-contained paper. Thus, in the next subsections we recall the equations and properties obtained from the application of Pontryagin's principle and some known results concerning the optimality of the IOI strategy.

For our purposes it is enough to use the version of the Pontryagin's Maximum Principle (PMP) stated and proved in [14]. Recall that the principle gives a first order necessary conditions for continuous-time optimal problems. Its geometric formulation takes place on the cotangent bundle of the state space. 
In our particular case, the (PMP) for the affine control system (2.3) on $\mathbb{R}^{n+2}$ reads: If $\boldsymbol{u}_{*}=\left(u_{1_{*}}, u_{2_{*}}\right)$ is an optimal control and $\mathbf{z}_{*}$ is the associated trajectory of $(2.3)$ on $\mathbb{R}^{n+2}$, there exists a constant $\lambda_{0} \geq 0$ and an absolutely continuous function $\boldsymbol{\lambda}:[0, T] \longrightarrow$ $\left(\mathbb{R}^{n+2}\right)^{*}, \quad \tau \mapsto \boldsymbol{\lambda}(\tau)=\left(\lambda(\tau), \lambda_{n+1}(\tau), \lambda_{n+2}(\tau)\right)$ with $\lambda(\tau)=\left(\lambda_{1}(\tau), \lambda_{2}(\tau), \cdots, \lambda_{n}(\tau)\right)$ such that for almost every $\tau \in \operatorname{Dom}\left(\mathbf{z}_{*}\right),\left(\boldsymbol{\lambda}, \lambda_{0}\right)$ never vanishing and the extremals satisfy

$$
\begin{aligned}
\dot{\mathbf{z}} & =\frac{\partial \mathcal{H}}{\partial \boldsymbol{\lambda}}\left(\mathbf{z}, \boldsymbol{\lambda}, u_{1}, u_{2}\right), \\
\dot{\boldsymbol{\lambda}} & =-\boldsymbol{\lambda}\left(u_{1} \mathcal{J} G_{1}+u_{2} \mathcal{J} G_{2}\right),
\end{aligned}
$$

Futhermore, the optimal control $\boldsymbol{u}_{*}$ minimizes the Hamiltonian

$$
\mathcal{H}=u_{1} \mathcal{H}_{G_{1}}+u_{2} \mathcal{H}_{G_{2}}+u_{1} \lambda_{0}
$$

over the set of admissible controls, through the curve $(\boldsymbol{\lambda}(\tau), \mathbf{z}(\tau))$. Here $\mathcal{H}_{G_{i}}(\mathbf{z}, \boldsymbol{\lambda})=$ $\left\langle\boldsymbol{\lambda}(\tau), G_{i}(\mathbf{z}(\tau))\right\rangle, i=1,2$ are the Hamiltonian lifts corresponding to each vector field $G_{i}$ respectively. Throughout this paper we assume $\lambda_{0}=1$, which corresponds to the normal extremals.

It is well know that the Poisson bracket of the Hamiltonian functions $\mathcal{H}_{G_{1}}$ and $\mathcal{H}_{G_{2}}$, denote by $\{\cdot, \cdot\}$, is associated with the Lie bracket by the relation

$$
\left\{\mathcal{H}_{G_{1}}, \mathcal{H}_{G_{2}}\right\}=\left\langle\boldsymbol{\lambda},\left[G_{1}, G_{2}\right]\right\rangle
$$

where, and the Lie bracket is defined to be $[X, Y](\mathbf{z})=\mathcal{J} Y(\mathbf{z})(X(\mathbf{z}))-\mathcal{J} X(\mathbf{z})(Y(\mathbf{z}))$ for any pair of vector fields $X$ and $Y$. Here $\mathcal{J}$ stand for the Jacobian differential operator with respect to $\mathbf{z}$, and $\langle\cdot, \cdot\rangle$ is the Euclidean inner product in $\mathbb{R}^{n+2}$ (see for instance, [1]).

Now, introducing the auxiliary variables $\widetilde{\lambda_{i}}=\lambda_{i}-\lambda_{n+1}$, the adjoint system (3.2) become the following dynamical systems

$$
\frac{d \widetilde{\lambda}}{d \tau}=A(\tau) \widetilde{\lambda}, \quad \widetilde{\lambda_{i}}(T)=-1, i=1, \cdots, n .
$$

where $\tilde{\lambda}=\left(\lambda_{1}-\lambda_{n+1}, \lambda_{2}-\lambda_{n+1}, \cdots, \lambda_{n}-\lambda_{n+1}\right)$ and $A(\tau)$ is a the $n \times n$ time dependent matrix, given by

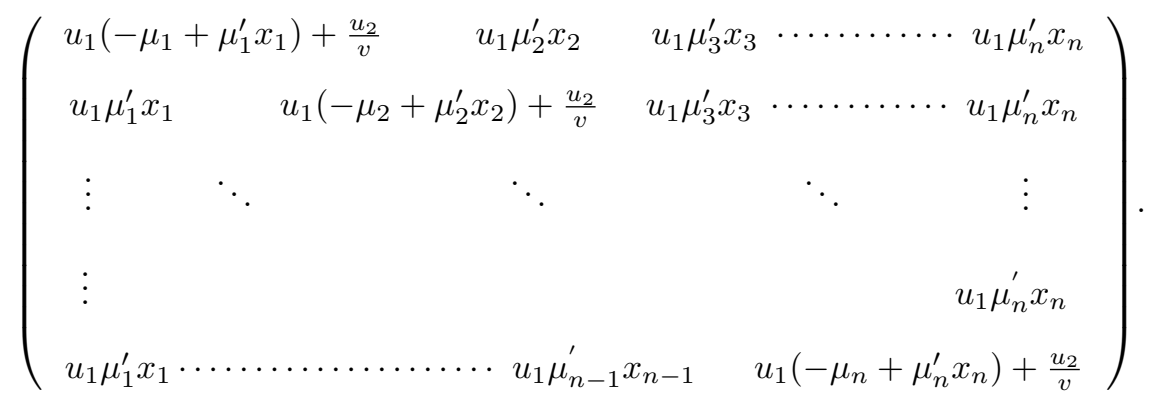

Consequently, one has 


$$
\widetilde{\lambda}(\tau) \neq 0 \text { for any } \tau \in\left[\tau_{0}, T\right] .
$$

Note that the Hamiltonian (3.3) can be equivalently written as follows

$$
\mathcal{H}=u_{1} \phi_{u_{1}}(\mathbf{z}, \boldsymbol{\lambda})+u_{2} \phi_{u_{2}}(\mathbf{z}, \boldsymbol{\lambda})
$$

where

$$
\left\{\begin{array}{l}
\phi_{u_{1}}(\mathbf{z}(\tau), \boldsymbol{\lambda}(\tau))=1+\sum_{j=1}^{n} \widetilde{\lambda}_{j}(\tau) \mu_{j}(s(\tau)) x_{j}(\tau), \\
\phi_{u_{2}}(\mathbf{z}(\tau), \boldsymbol{\lambda}(\tau))=\lambda_{n+2}+\lambda_{n+1} \frac{\left(s_{i n}-s\right)}{v}-\frac{1}{v} \sum_{j=1}^{n} \lambda_{j} x_{j}
\end{array}\right.
$$

For notationally simplicity, we now write $\phi_{u_{i}}(\tau)$ for $\phi_{u_{1}}(\mathbf{z}(\tau), \boldsymbol{\lambda}(\tau))$.

In the rest of the paper we will consider only increasing growth functions:

Assumption A1. The functions $\mu_{i}(\cdot)$ are non decreasing.

Lemma 3.1 Under Assumption A1, the following assertions hold:

(i) The matrix $A(\tau)$ has non-negative off-diagonal terms, i.e., the dynamical system (3.5) is cooperative.

(ii) The vector $m^{1}(\cdot)$, defined by

$$
m^{1}(\tau)=\left(\begin{array}{c}
\mu_{1}^{\prime}(s(\tau)) x_{1}(\tau) \\
\vdots \\
\mu_{n}^{\prime}(s(\tau)) x_{n}(\tau)
\end{array}\right) .
$$

lies in $\mathbb{R}_{+}^{n}$.

Proof: The proof is direct and it was already established in [11].

Notice that the controls are not obviously determined by the minimum condition at times $\tau$ when $\phi_{u_{1}}(\tau)=\phi_{u_{2}}(\tau)=0$. Indeed, PMP is not able to distinguish minima from maxima in that periods of time. However, it is well known from the optimal control theory that an optimal trajectory may well be singular; this is, switching functions $\phi_{u_{i}}(\tau), i=1,2$ may vanish identically along the trajectory. The characterization of such trajectories, is in general a difficult task, but we provide a context for which this holds for our problem defining the value function in (2.5).

\section{Singular trajectories}

In our context, a singular trajectory or singular arc corresponds to an extremal curve for which there exists a nontrivial interval $\left[\tau_{1}, \tau_{2}\right] \subset[0, T]$ where both switching functions $\phi_{u_{i}}(\tau)$ are identically zero. This can be understood as an extension of the standard definition of singular arcs when only one control is considered (e.g. [7, Part III, Ch. 2]).

Since the switching functions play a crucial role in the definition of a singular arc, we proceed here below to analyze them. In particular, we focus on the computation of their derivatives. 
Lemma 4.1 For dynamics (2.2), the first derivative with respect to the time $\tau$ of the switching functions $\phi_{u_{i}}(\tau), i=1,2$, defined in (3.8), are given by

$$
\left\{\begin{aligned}
\frac{d \phi_{u_{2}}}{d \tau} & =-u_{1} \frac{\left(s_{i n}-s\right)}{v}\left\langle\widetilde{\lambda}, m^{1}\right\rangle, \\
\frac{d \phi_{u_{1}}}{d \tau} & =u_{2} \frac{\left(s_{i n}-s\right)}{v}\left\langle\widetilde{\lambda}, m^{1}\right\rangle .
\end{aligned}\right.
$$

where $\tilde{\lambda}=\left(\lambda_{1}-\lambda_{n+1}, \lambda_{2}-\lambda_{n+1}, \cdots, \lambda_{n}-\lambda_{n+1}\right)$ and $m^{1}=m^{1}(\tau)$ was defined in (3.9).

Proof: This property was shown in [11, Eq. (6.5)].

Denote by

$$
\psi:=\frac{\left(s_{i n}-s\right)}{v}\left\langle\widetilde{\lambda}, m^{1}\right\rangle
$$

the function appearing in the first derivative of the switching functions. We now establish a formula of the $\mathrm{n}$-order derivative for $\psi$.

Proposition 4.2 Consider a fixed time $\tau$. For a given integer $j$, define $m^{j}=m^{j}(\tau)$ by

$$
m^{j}(\tau)=\left(\begin{array}{c}
\mu_{1}^{(j)}(s(\tau)) x_{1}(\tau) \\
\vdots \\
\mu_{n}^{(j)}(s(\tau)) x_{n}(\tau)
\end{array}\right),
$$

where $\mu_{i}^{(j)}$ denotes the $j$-th derivative of $\mu_{i}$ with $i=1,2, \cdots, n$. Then if $\left\langle\widetilde{\lambda}, m^{k}\right\rangle=0$ for every $k=1, \cdots, j$, we have

$$
\frac{d^{j} \psi}{d \tau^{j}}=\frac{\left(s_{i n}-s\right)}{v}\left\langle\widetilde{\lambda}, m^{j+1}\right\rangle\left(\frac{d s}{d \tau}\right)^{j}
$$

Proof: We proceed to prove the desired implication by induction on $j$. The case $j=1$ follows directly from the definition of $\psi$ given in (4.2). Now, suppose that the induction hypothesis holds true for a given $j$, that is, the following assertion is fulfilled: if $\left\langle\widetilde{\lambda}, m^{k}\right\rangle=0$ for every $k=1, \cdots, j$ then (4.4) is verified. In order to establish our induction argument, we also suppose that $\left\langle\widetilde{\lambda}, m^{k}\right\rangle=0$ for every $k=1, \cdots, j$, and we proceed to prove that equation (4.4) holds true when we replace $j$ by $j+1$.

The induction hypothesis implies that (4.4) holds, this yield

$$
\frac{d^{j+1} \psi}{d \tau^{j+1}}=\frac{\left(s_{i n}-s\right)}{v}\left(\frac{d s}{d \tau}\right)^{j} \frac{d}{d \tau}\left\langle\widetilde{\lambda}, m^{j+1}\right\rangle+\frac{d}{d \tau}\left(\frac{\left(s_{i n}-s\right)}{v}\left(\frac{d s}{d \tau}\right)^{j}\right)\left\langle\widetilde{\lambda}, m^{j+1}\right\rangle
$$

On the other hand, direct computations lead to

$$
\left\langle\widetilde{\lambda}, A(\tau)^{\top} m^{j+1}\right\rangle=\frac{u_{2}}{v}\left\langle\widetilde{\lambda}, m^{j+1}\right\rangle+u_{1}\left(\sum_{i} \mu_{i}^{(j+1)} x_{i}\right)\left\langle\widetilde{\lambda}, m^{1}\right\rangle-u_{1}\left\langle\widetilde{\lambda}, \widetilde{m}^{j+1}\right\rangle
$$


and

$$
\left\langle\widetilde{\lambda}, \frac{d}{d \tau} m^{j+1}\right\rangle=\left(\frac{d s}{d \tau}\right)\left\langle\widetilde{\lambda}, m^{j+2}\right\rangle-\frac{u_{2}}{v}\left\langle\widetilde{\lambda}, m^{j+1}\right\rangle+u_{1}\left\langle\widetilde{\lambda}, \widetilde{m}^{j+1}\right\rangle,
$$

where we have used the auxiliary notation

$$
\widetilde{m}^{j}=\left(\begin{array}{c}
\mu_{1}^{(j)} \mu_{1} x_{1} \\
\vdots \\
\mu_{n}^{(j)} \mu_{n} x_{n}
\end{array}\right) .
$$

So, equations (4.6) and (4.7) lead to

$$
\begin{aligned}
\frac{d}{d \tau}\left\langle\widetilde{\lambda}, m^{j+1}\right\rangle & =\left\langle\widetilde{\lambda}, A(\tau)^{\top} m^{j+1}\right\rangle+\left\langle\widetilde{\lambda}, \frac{d m^{j+1}}{d \tau}\right\rangle \\
& =\frac{d s}{d \tau}\left\langle\widetilde{\lambda}, m^{j+2}\right\rangle+u_{1}\left(\sum_{i=1}^{n} \mu_{i}^{(j+1)} x_{i}\right)\left\langle\widetilde{\lambda}, m^{1}\right\rangle,
\end{aligned}
$$

Replacing (4.8) into (4.5) we have

$$
\begin{aligned}
\frac{d^{j+1} \psi}{d \tau^{j+1}}=\frac{\left(s_{i n}-s\right)}{v}\left(\frac{d s}{d \tau}\right)^{j+1}\left\langle\widetilde{\lambda}, m^{j+2}\right\rangle & +u_{1}\left(\frac{d s}{d \tau}\right)^{j}\left(\sum_{i=1}^{n} \mu_{i}^{(j+1)} x_{i}\right)\left\langle\widetilde{\lambda}, m^{1}\right\rangle \\
& +\frac{d}{d \tau}\left[\frac{\left(s_{i n}-s\right)}{v}\left(\frac{d s}{d \tau}\right)^{j}\right]\left\langle\widetilde{\lambda}, m^{j+1}\right\rangle,
\end{aligned}
$$

Finally, since by hypothesis the two last terms in the right hand side of (4.8) are equal to zero, it follows that

$$
\frac{d^{j+1} \psi}{d \tau^{j+1}}=\frac{\left(s_{i n}-s\right)}{v}\left(\frac{d s}{d \tau}\right)^{j+1}\left\langle\widetilde{\lambda}, m^{j+2}\right\rangle,
$$

which proves our induction argument.

Now we are ready to state the main result of this section.

Theorem 4.3 Consider initial condition $\xi=\left(x_{10}, \ldots, x_{n 0}, s_{0}, v_{0}\right) \in \mathcal{D}$. Suppose that the determinant of the matrix

$$
\mathfrak{D}=\left(\begin{array}{ccc}
\mu_{1}^{(1)}(s) & \cdots & \mu_{n}^{(1)}(s) \\
\mu_{1}^{(2)}(s) & \cdots & \mu_{n}^{(2)}(s) \\
\vdots & \ddots & \vdots \\
\mu_{1}^{(n)}(s) & \cdots & \mu_{n}^{(n)}(s)
\end{array}\right)
$$

is nonsingular for any $s \in\left(0, s_{i n}\right)$. Here $\mu_{i}^{(j)}$ denotes the $j$-th derivative of function $\mu_{i}$. Then, an extremal curve is a singular arc on $\left(\tau_{1}, \tau_{2}\right) \subset[0, T]$ if and only if $s(\cdot)$ is constant on $\left(\tau_{1}, \tau_{2}\right)$. 
Proof: First note that an initial condition $\xi=\left(x_{10}, \ldots, x_{n 0}, s_{0}, v_{0}\right) \in \mathcal{D}$ implies that $s(\tau) \in\left(0, s_{i n}\right)$ for all time $\tau$, so we will always suppose that $s$ will remain in this interval.

Suppose that a singular arc defined in an interval of time $\left(\tau_{1}, \tau_{2}\right) \subset[0, T]$ is optimal. Since $u_{1}$ and $u_{2}$ are not simultaneously equal to zero, $\left\langle\widetilde{\lambda}, m^{1}\right\rangle=0$ on $\left(\tau_{1}, \tau_{2}\right)$ (via equations (4.1)); this in particular implies that the derivatives of $\psi$ with respect to the time $\tau$ are null on $\left(\tau_{1}, \tau_{2}\right)$. So, we can apply inductively Proposition 4.2 and obtain that

$$
\frac{d^{j} \psi}{d \tau^{j}}=\frac{\left(s_{i n}-s\right)}{v}\left(\frac{d s}{d \tau}\right)^{j}\left\langle\widetilde{\lambda}, m^{j+1}\right\rangle=0
$$

on $\left(\tau_{1}, \tau_{2}\right)$, and for all $j=1,2, \cdots, n-1$.

Suppose now that $\frac{d s}{d \tau} \neq 0$. It follows from (4.11) that

$$
\left\langle\widetilde{\lambda}, m^{j}\right\rangle=0, \quad j=1, \cdots, n \text { on }\left(\tau_{1}, \tau_{2}\right) .
$$

Hence, using the fact that $\tilde{\lambda}(\tau) \neq 0$ for all $\tau$ (see (3.6)), we have that $m^{1}, m^{2}, \cdots, m^{n}$ are linearly dependent for any $\tau \in\left(\tau_{1}, \tau_{2}\right)$. However, we have $m^{j}=X \eta^{j}$ with

$$
X=\left(\begin{array}{ccc}
x_{1} & & \\
& \ddots & \\
& & x_{n}
\end{array}\right) \text { and } \eta^{j}=\left(\begin{array}{c}
\mu_{1}^{(j)}(s(\tau)) \\
\vdots \\
\mu_{n}^{(j)}(s(\tau))
\end{array}\right) \text {, }
$$

And, since $X$ is clearly non-singular and the vectors $\eta^{j}$ are linearly independent (because they are the rows of the matrix $\mathfrak{D}$, which is non-singular), these is a contradiction with the linear dependence of $m^{j}$, concluding thus $\frac{d s}{d \tau}=0$.

Reciprocally, suppose now $\frac{d s}{d \tau}=0$ on $\left(\tau_{1}, \tau_{2}\right)$. Equation (4.8) (with $j=0$ for which the formula is still valid) leads to

$$
\frac{d}{d \tau}\langle\widetilde{\lambda}, m\rangle=\varphi(\tau)\langle\widetilde{\lambda}, m\rangle,
$$

for some given function $\varphi(\cdot)$. Hence, $\langle\widetilde{\lambda}, m\rangle=C \exp \left(\int_{0}^{\tau} \varphi(\tau) d \tau\right)$ on $\left(\tau_{1}, \tau_{2}\right)$, for a real constant $C$.

Suppose now that $C \neq 0$. One obtains from (4.1) that the switching functions $\phi_{u_{1}}$ and $\phi_{u_{2}}$ are both monotone on $\left(\tau_{1}, \tau_{2}\right)$. This implies that $\phi_{u_{1}}$ and $\phi_{u_{2}}$ are both strictly positive on $\left(\tau_{1}, \tau_{2}\right)$. Consequently, (3.7) implies that controls $u_{1}$ and $u_{2}$ are both equal to zero. This is a contradiction.

Therefore, the only possibility is $C=0$. In this case, (4.1) implies that the switching functions $\phi_{u_{1}}$ and $\phi_{u_{2}}$ are both constant on $\left(\tau_{1}, \tau_{2}\right)$. Since $u_{1}$ and $u_{2}$ are strictly positive, it follows from Lemma 4.1 that $\phi_{u_{1}}=\phi_{u_{2}}=0$ on $\left(\tau_{1}, \tau_{2}\right)$. The theorem follows.

Assumption A2. The determinant of $\mathfrak{D}$ given in (4.10) is non null.

Remark 3 The Assumption A2 extends to an arbitrary number of species the also called Assumption A2 in [11] established only for two species. 


\subsection{The n-species case with a Monod growth function}

In section we analyze the case when the growth function of each species of microorganism considered in the SBR follows a Monod law growth function [8], that is

$$
\mu_{i}(s)=\frac{\mu_{\max , i} s}{K_{i}+s}, \quad i=1, \cdots, n
$$

where, for the $i$-species, $\mu_{\max , i}$ and $K_{i}$ stand for the maximal growth rate and for the half saturation constant or Monod constant. As a first step, we establish a formula for the derivatives of a general Monod function.

Lemma 4.4 For any Monod function described by (4.14), its derivatives with respect to $s$ are given by

$$
\mu^{(j)}(s)=\frac{(-1)^{j+1} j ! \mu_{\max , i}}{(K+s)^{j+1}}, \quad j=1,2, \cdots, n
$$

Proof: The proof follows directly from induction on $j$.

As an application of the results obtained in this section, a characterization of singular arcs, for the case in which several species compete for the same substrate and all of them have Monod growth function, is given in the following proposition.

Proposition 4.5 Assume that any growth function in our setting follows a Monod law, which is different from the growth function of any other species. This is, if

$$
\mu_{i}(s)=\frac{\mu_{\max , i} s}{K_{i}+s}, \quad i=1, \cdots, n
$$

and $K_{i} \neq K_{j}$, for all $i \neq j$. Then, an extremal curve is a singular arc on $\left(\tau_{1}, \tau_{2}\right) \subset[0, T]$ if and only if $s(\cdot)$ is constant on $\left(\tau_{1}, \tau_{2}\right)$.

Proof: Lemma 4.4 and some additional computations show that the determinant of the matrix $\mathfrak{D}$, defined in (4.10), is given by

$$
\frac{n\left([(n-1) !]^{2}\right)\left(\prod_{l=1}^{n} \mu_{\max , l}\right)\left(\prod_{l=1}^{n} K_{l}\right)\left(\prod_{i, j=1, i>j}^{n}\left(K_{i}-K_{j}\right)\right)}{\prod_{l=1}^{n}\left(K_{l}+s\right)^{n+1}}
$$

Now, since $K_{i} \neq K_{j}$, for all $i, j=1, \cdots, n$ it follows that the determinant of $\mathfrak{D}$ is non null, and consequently $\mathfrak{D}$ is nonsingular. Then, the desired result follows from Theorem 4.3.

\section{The Immediate One Impulse strategy}

This section is devoted to evaluate whenever the Immediate One Impulse strategy (denoted from now on by $I O I$ ) is optimal for our minimal time control problem, when one species is highly performant and the others all are sufficiently close one to each other. We begin by first describing the $I O I$ strategy. 
From an initial state $\xi=\left(x_{10}, \ldots, x_{n 0}, s_{0}, v_{0}\right) \in \mathcal{D}$ at time $t_{0}$. We define the $I O I$ consisting of making:

1. An impulse of volume $v_{\max }-v_{0}$ at $t_{0}$. This can be achieved by $r(\tau)=0, u(\tau)=$ $u_{\max }$, for $\tau \in\left[t_{0}, t_{0}+\left(v_{\max }-v_{0}\right) / u_{\max }\right]$.

2. A null control (no feeding) until the concentration $s(\tau)$ reaches $s_{\text {out }}$.

For convenience, we shall denote by $\tilde{x}_{i 0}(\xi), i=1, \cdots, n$ and $\tilde{s}_{0}(\xi)$ the concentrations obtained with an impulse of volume $v_{\max }-v_{0}$ from a state $\xi=\left(x_{10}, \ldots, x_{n 0}, s_{0}, v_{0}\right) \in \mathcal{D}$ :

$$
\tilde{x}_{i 0}(\xi)=x_{i 0} \frac{v_{0}}{v_{\max }}, i=1, \cdots, n \quad, \quad \tilde{s_{0}}(\xi)=s_{0} \frac{v_{0}}{v_{\max }}+s_{i n}\left(1-\frac{v_{0}}{v_{\max }}\right) .
$$

Notice that for the particular case $\tilde{s}_{0}(\xi) \leq s_{\text {out }}$, the first step only is used.

We consider a family of functions $\varphi_{c}(\cdot)$ defined on $\left(\mathbb{R}_{+}^{n} \backslash\{0\}\right) \times \mathbb{R}_{+}$and parameterized by $c>0$ :

$$
\varphi_{c}\left(x_{10}, \ldots, x_{n 0}, s_{0}\right)=\inf \left\{t-t_{0} \mid s^{t_{0}, x_{10}, \ldots, x_{n 0}, s_{0}}(t) \leq c\right\},
$$

where $\left(s^{t_{0}, x_{10}, \ldots, x_{n 0}, s_{0}}\right)(\cdot)$ is solution of the free dynamics:

$$
\left\{\begin{array}{l}
\frac{d x_{i}}{d \tau}=\mu_{i}(s) x_{i}, \quad x_{i}\left(t_{0}\right)=x_{i 0} \quad(i=1 \cdots n), \\
\frac{d s}{d \tau}=-\sum_{i=1}^{n} \mu_{i}(s) x_{i}, \quad s\left(t_{0}\right)=s_{0} .
\end{array}\right.
$$

Standard analysis of minimal time problems shows that $\varphi_{c}(\cdot)$ are Lipschitz-continuous functions and solutions, in the viscosity sense, of the partial differential equation (see for instance [10])

$$
\sum_{j=1}^{n}\left(\partial_{x_{j 0}} \varphi_{c}\left(x_{10}, \ldots, x_{n 0}, s_{0}\right)-\partial_{s_{0}} \varphi_{c}\left(x_{10}, \ldots, x_{n 0}, s_{0}\right)\right) \mu_{j}\left(s_{0}\right) x_{j 0}+1=0,
$$

on the domain $\left(\mathbb{R}_{+}^{n} \backslash\{0\}\right) \times(c,+\infty)$ with boundary conditions

$$
\varphi_{c}\left(., s_{0}\right)=0, \quad \forall s_{0} \in(0, c] .
$$

The time cost of the IOI strategy can then be simply written in terms of above functions, as follows

$$
T_{I O I}(\xi)=\varphi_{s_{\text {out }}}\left(\tilde{x}_{10}(\xi), \ldots, \tilde{x}_{n 0}(\xi), \tilde{s}_{0}(\xi)\right),
$$

where $\left(\tilde{x}_{10}(\xi), \ldots, \tilde{x}_{n 0}(\xi)\right)$ and $\tilde{s}_{0}(\xi)$ are given by $(5.1)$.

Now, we assume the following regularity on the function $\varphi_{c}(\cdot)$,

Assumption A3. For any $c>0$, the function $\varphi_{c}(\cdot)$ is $C^{1}$ on $\left(\mathbb{R}_{+}^{n} \backslash\{0\}\right) \times(c,+\infty)$. 
Remark 4 Observe that the sub-optimal IOI strategy has a finite time cost $T_{I O I}(\xi)$ for any initial condition $\xi$ in the domain $\mathcal{D}$. Consequently, the optimal value $V(\xi)$ is finite for any $\xi$ in $\mathcal{D}$.

Finally, Proposition 5.3 in [11] provides a direct way to verify when the IOI strategy is optimal for any initial condition $\xi=\left(x_{10}, \ldots, x_{n 0}, s_{0}, v_{0}\right) \in \mathcal{D}$. We recall here below this key tool.

Theorem 5.1 Under Assumption A3, the one impulse strategy is optimal for any $\xi \in \mathcal{D}$ if and only if

$$
\Delta_{u_{1}} T_{I O I}(\xi) \geq 0, \forall \xi \in \mathcal{D} \text { s.t. } T_{I O I}(\xi)>0 .
$$

where $\Delta_{u_{1}} T_{I O I}(\xi)$ is computed as follows

$$
\begin{aligned}
& \Delta_{u_{1}} T_{I O I}(\xi)= \\
& \quad \sum_{j=1}^{n}\left(\partial_{x_{j 0}} \varphi_{s_{\text {out }}}\left(\tilde{x}_{10}(\xi), \cdots, \tilde{x}_{n 0}(\xi), \tilde{s}_{0}(\xi)\right)\right)-\partial_{s_{0}} \varphi_{s_{\text {out }}}\left(\tilde{x}_{10}(\xi), \cdots, \tilde{x}_{n 0}(\xi), \tilde{s}_{0}(\xi)\right) \\
& \quad \times \widetilde{x}_{j 0}\left(\mu_{j}\left(s_{0}\right)-\mu_{j}\left(\widetilde{s}_{0}(\xi)\right)\right)
\end{aligned}
$$

In order to evaluate whenever the IOI strategy is optimal, the following assumption will be crucial in the sequel.

Assumption A4 For any $s_{1}, s_{2} \in\left[s_{\text {out }}, s_{i n}\right]$ and $j=1, \cdots, n-1$, one has

$$
s_{2} \geq s_{1} \Rightarrow \mu_{n}\left(s_{2}\right) \mu_{j}\left(s_{1}\right) \geq \mu_{j}\left(s_{2}\right) \mu_{n}\left(s_{1}\right) .
$$

Remark 5 Notice that for Monod laws of the form (4.14), implications (5.8) are exactly fulfilled when $K_{n} \geq K_{j}, j=1, \cdots, n-1$. Indeed, direct computations lead to

$$
\mu_{n}\left(s_{2}\right) \mu_{j}\left(s_{1}\right)-\mu_{j}\left(s_{2}\right) \mu_{n}\left(s_{1}\right)=\frac{\mu_{\max , n} \mu_{\max , 1} s_{1} s_{2}\left(s_{2}-s_{1}\right)\left(K_{n}-K_{j}\right)}{\left(K_{j}+s_{2}\right)\left(K_{j}+s_{1}\right)\left(K_{n}+s_{2}\right)\left(K_{n}+s_{1}\right)} .
$$

In [11] it is shown that, when only two species coexist and one is clearly more performant than other one (which is reflected via the condition $\mu_{2}(s) \geq \mu_{1}(s)$, for all $s \in\left(0, s_{s i n}\right]$, on the growth functions), then the IOI strategy is optimal. Moreover, numerical experiences proved that this condition is also necessary. In other words, the fact that growth functions are increasing is not enough to ensure the optimality of the IOI strategy. For instance, see example below.

Following this idea of our analysis, one would like to investigate if similar behaviors occur in this case when more than two species coexist $(n>2)$. For this, in what follows, we assume the existence of a species which is clearly more performant than the other ones. This is stated via the following assumption.

Assumption $\mathbf{A 5} \mu_{n}(s) \geq \mu_{j}(s), \forall j=1, \cdots, n-1, \forall s \in\left(0, s_{i n}\right]$. 
In this framework, next numerical example shows that Assumptions $A 1, A 3, A 4$ and $A 5$ do not ensure the optimality of the strategy IOI. In other words, we establish the surprising result that in the case of more than two species, even the existence of a "most performant species" is not enough for this purpose. Next example shows that, even under the Assumptions A1, A3, A4 and A5', strategy IOI is not necessarily optimal.

Example: We consider the following three growth functions (see Figure 1):

$$
\mu_{1}(s)=\frac{s}{1+s}, \mu_{2}(s)=\frac{2 s}{1.5+s}, \mu_{3}(s)=\frac{4 s}{2+s} .
$$

They clearly fulfill Assumptions $A 1, A 2, A 3, A 4$ and $A 5$.

Consider also the parametric values $v_{\max }=10, s_{\text {out }}=0.1$ and $s_{\text {in }}=5$, and the initial conditions $x_{10}=1, x_{20}=0.001, s_{0}=3$ and $v_{0}=1$. In table 1 we compare, for different values of the initial conditions $x_{30}$, the time achieved by the strategy IOI with the time achieved by an alternative strategy consisting of to reach, as fast as possible, a given level $s^{*}$ in $\left(s_{\text {out }}, s_{i n}\right)$, then to keep $s$ constant and equal to $s^{*}$ until $v$ reaches $v_{\max }$, and finally, put $u=0$ and $r=1$ (which means to close $S A\left(s^{*}\right)$, and the level $s^{*}$ is numerically computed in order to minimize the cost of this type of strategies among all possible values of $s^{*}$ in $\left(s_{\text {out }, s_{i n}}\right)$. Recall that in this case of two species, it was shown in [11] that this strategy besides $I O I$ when Assumptions $A 1, A 2, A 3, A 4$ and $A 5$ were fulfilled.

So, reported results in Table 1 establish that $S A\left(s^{*}\right)$ has an improvement close to $25 \%$ with respect to $I O I$ for some values of $x_{30}$. We thus conclude that IOI is not optimal for this particular setting.

Tabla 1.

\begin{tabular}{|c||c|c|c|c|}
\hline$x_{30}$ & $T(I O I)$ & $s^{*}$ & $T\left(S A\left(s^{*}\right)\right)$ & gain \\
\hline $10^{-4}$ & 5.416174 & 4.226000 & 5.402767 & $0.2 \%$ \\
$10^{-3}$ & 5.389022 & 3.540000 & 4.978126 & $7.6 \%$ \\
$10^{-2}$ & 5.172141 & 3.442000 & 4.170769 & $19.4 \%$ \\
0.05 & 4.669824 & 3.344000 & 3.548414 & $24.0 \%$ \\
0.1 & 4.350146 & 3.246000 & 3.274169 & $24.7 \%$ \\
0.5 & 3.458854 & 3.050000 & 2.620281 & $24.2 \%$ \\
\hline
\end{tabular}

The latter reveals another surprising and interesting result. Note that growth functions satisfy the next stronger condition than $A 5$ :

Assumption A5' $\mu_{n}(s) \geq \mu_{n-1}(s) \geq \cdots \geq \mu_{1}(s), \forall s \in\left(0, s_{i n}\right]$.

This means that the performance of all the species are perfectly ordered. Thus Assumption A5' can be interpreted as a stronger extension of the corresponding condition in [11]. However, the example above states that even under this stronger condition it is not possible to ensure the optimality of the IOI strategy. 


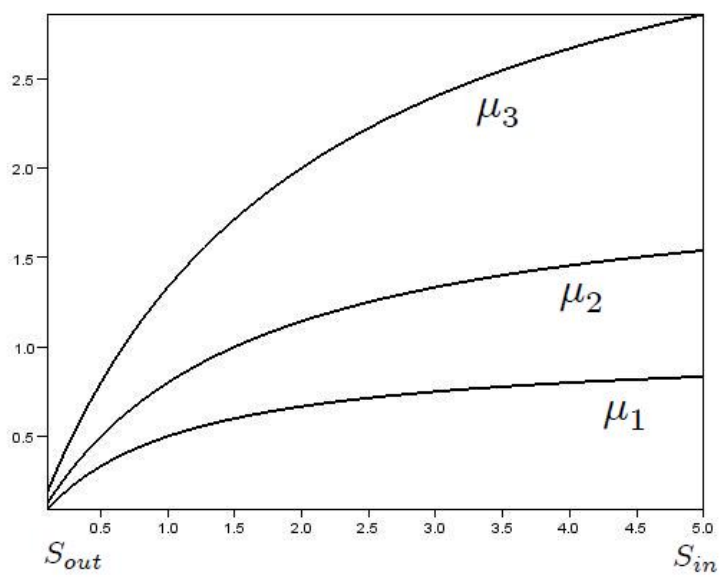

Figure 1: Graphs of the three growth functions considered in the example.

We proceed to investigate other hypotheses (stronger than A5) for which one can prove that IOI strategy is optimal. For this, at this stage of our analysis, we need to state a technical lemma for the family of functions $\varphi_{c}(\cdot)$. This is direct extensions of the corresponding results in [11]. Since its proof is very similar to those appearing in [11], it is omitted here.

Lemma 5.2 Under Assumptions A1, A3, A4 and A5, functions $\varphi_{c}(\cdot)$ posses the following properties for all $c \in\left(0, s_{i n}\right),(y, z):=\left(x_{10}, \ldots, x_{n 0}, s_{0}\right) \in\left(\mathbb{R}_{+}^{2} \backslash\{0\}\right) \times\left(c, s_{i n}\right]$ and $i=1, \cdots, n-1$ :

$$
\begin{aligned}
\text { i. } \partial_{z} \varphi_{c}(y, z) & \geq \partial_{x_{n 0}} \varphi_{c}(y, z), \\
\text { ii. } \quad \frac{\mu_{n}(z)-\mu_{n}(\tilde{z})}{\mu_{n}(\tilde{z})} & \leq \frac{\mu_{i}(z)-\mu_{i}(\tilde{z})}{\mu_{i}(\tilde{z})} \leq 0 .
\end{aligned}
$$

Now, we are in position to establish an additional condition for which IOI is optimal in this framework.

Theorem 5.3 Suppose that the Assumptions A1, A3, A4 and A5 are fulfilled. Suppose also that growth functions $\mu_{i}$, for $i=1, \ldots, n-1$, are all close enough one each other on $\left[0, s_{i n}\right]$, that is, there exists a small enough $\epsilon>0$ such that for all $i, j=1, \ldots, n-1$, $s \in\left[0, s_{\text {in }}\right]$ one has

$$
\mu_{i}(s)-\mu_{j}(s) \leq \epsilon .
$$

Then IOI strategy is optimal for any initial condition in $\mathcal{D}$.

Proof: Consider $\xi=\left(x_{10}, \ldots, x_{n 0}, s_{0}, v_{0}\right):=\left(y, z, v_{0}\right) \in \mathcal{D}$ such that $T_{I O I}(\xi)>0$. Let 
us denote for all $i=1, \ldots, n-1$ :

$$
\gamma_{i}=\left(\frac{\mu_{i}(z)-\mu_{i}(\tilde{z})}{\mu_{i}(\tilde{z})}\right) / \sum_{j=1}^{n-1} \frac{\mu_{j}(z)-\mu_{j}(\tilde{z})}{\mu_{j}(\tilde{z})} .
$$

Part iv of Lemma 5.2 ensures that $\gamma_{i} \geq 0$ for all $i$. Moreover, Hypothesis (5.9) implies that

$$
\gamma_{i} \in\left[\frac{1}{n-1}-\tilde{\epsilon}, \frac{1}{n-1}+\tilde{\epsilon}\right], \quad \forall i=1, \ldots, n-1,
$$

for some $\tilde{\epsilon}>0$. Note that the magnitude of $\tilde{\epsilon}$ is driven by $\epsilon$, that is, $\tilde{\epsilon}$ can be set as small as necessary provided that $\epsilon$ is choosen small enough. Indeed, it suffices to note that $\mu_{i}(\tilde{z}) \geq \mu_{i}(z)$ and the fact that the $\mu_{i}$ are bounded on $\left[0, s_{i n}\right]$.

Notice that the term $\Delta_{u_{1}} T_{I O I}(\xi)$, given in (5.7), can be written as follows

$$
\Delta_{u_{1}} T_{I O I}(\xi)=\sum_{j=1}^{n}\left(\partial_{y_{j}} \varphi_{s_{\text {out }}}(\tilde{y}, \tilde{z})-\partial_{z} \varphi_{\text {sout }_{\text {ou }}}(\tilde{y}, \tilde{z})\right) \mu_{j}(\tilde{z}) \tilde{y}_{j} \frac{\mu_{j}(z)-\mu_{j}(\tilde{z})}{\mu_{j}(\tilde{z})} .
$$

So, from Part iv of Lemma 5.2 and (5.10) we obtain that $\gamma_{n} \geq \gamma_{i} \geq \frac{1}{n-1}-\tilde{\epsilon}$, for all $i=1, \ldots, n-1$. Then, Part i of Lemma 5.2 implies

$$
\begin{aligned}
& \frac{\Delta_{u_{1}} T_{I O I}(\xi)}{\sum_{j=1}^{n-1} \frac{\mu_{j}(z)-\mu_{j}(\tilde{z})}{\mu_{j}(\tilde{z})}} \leq \sum_{j=1}^{n-1}\left(\partial_{y_{j}} \varphi_{s_{\text {out }}}(\tilde{y}, \tilde{z})-\partial_{z} \varphi_{s_{\text {out }}}(\tilde{y}, \tilde{z})\right) \mu_{j}(\tilde{z}) \tilde{y}_{j} \gamma_{j} \\
& +\left(\partial_{y_{n}} \varphi_{s_{\text {out }}}(\tilde{y}, \tilde{z})-\partial_{z} \varphi_{s_{\text {out }}}(\tilde{y}, \tilde{z})\right) \mu_{n}(\tilde{z}) \tilde{y_{n}}\left(\frac{1}{n-1}-\tilde{\epsilon}\right) \\
& \leq \frac{1}{n-1} \sum_{j=1}^{n}\left(\partial_{y_{j}} \varphi_{s_{\text {out }}}(\tilde{y}, \tilde{z})-\partial_{z} \varphi_{s_{\text {out }}}(\tilde{y}, \tilde{z})\right) \mu_{j}(\tilde{z}) \tilde{y}_{j} \\
& +\tilde{\epsilon} \sum_{j=1}^{n}\left|\partial_{y_{j}} \varphi_{s_{\text {out }}}(\tilde{y}, \tilde{z})-\partial_{z} \varphi_{s_{\text {out }}}(\tilde{y}, \tilde{z})\right| \mu_{j}(\tilde{z}) \tilde{y}_{j} \\
& =-\frac{1}{n-1}+\tilde{\epsilon} \sum_{j=1}^{n}\left|\partial_{y_{j}} \varphi_{s_{\text {out }}}(\tilde{y}, \tilde{z})-\partial_{z} \varphi_{\text {sout }_{\text {o }}}(\tilde{y}, \tilde{z})\right| \mu_{j}(\tilde{z}) \tilde{y}_{j}
\end{aligned}
$$

where this last equality is due to (5.4). Consequently

$$
\Delta_{u_{1}} T_{I O I}(\xi) \geq-\left(\sum_{j=1}^{n-1} \frac{\mu_{j}(z)-\mu_{j}(\tilde{z})}{\mu_{j}(\tilde{z})}\right)\left(\frac{1}{n-1}-\tilde{\epsilon} \sum_{j=1}^{n}\left|\partial_{y_{j}} \varphi_{s_{\text {out }}}(\tilde{y}, \tilde{z})-\partial_{z} \varphi_{\text {sout }_{\text {ou }}}(\tilde{y}, \tilde{z})\right| \mu_{j}(\tilde{z}) \tilde{y}_{j}\right)
$$

Therefore, since $y, \tilde{y}, z, \tilde{z}$ remain in a compact set and all the function involved are $C^{1}$, the right hand side term in the above expression is positive provided that $\tilde{\epsilon}$ is small enough. We thus conclude by Theorem 5.1. 


\section{References}

[1] Arnold V. I : Méthodes Mathématiques de Mécanique Classique, Editions Mir, Moscou, (1976).

[2] KREneR: The high order maximal principle and its application to singular extremals, SIAM J. Control Optim., 15, (2), (1997), 256-293.

[3] B. Bonnard And I. Kupka: Théorie des singularités de lápplication entrée/sortie et optimalité des trajectoires singuliéres dans le problemé du temps minimal, Forum Math., 5, (1993), 111-159.

[4] D'Ans G., D. Gottlieb and P. Kokotovic: Optimal control of bacterial growth, Automatica, 8, (1972), 729-736.

[5] A.J. Sussmann: Envelopes, higher order optimality conditions and Lie Brackets, I.E.E.E, Conf. Decision and Control, (1999).

[6] Irvine R. L. And L. H. Ketchum: Sequencing batch reactors for biological wastewater treatment, Critical Rev. Environ. Control, 18, (1989), 255-294.

[7] J.F. Bonnans, P. Rouchon: Commande et optimisation de systémes dynamiques, Éditions de l'École polytechnique, (2005).

[8] J. Monod : Recherches sur la croissance des cultures bactriennes, Paris, France: Hermes, (1942).

[9] J. Moreno: Optimal control of bioreactors for the wastewater treatment. Optimal Control, Applications and Methods, 20, (3), (1999), 145-164.

[10] Bardi M. And Capuzzo-Dolcetta: Optimal Control and Viscosity solutions of Hamilton-Jacobi-Bellman Equations, Birkhaüser, Boston, (1997).

[11] P. Gajardo, H. Ramírez, A. Rapaport: Minimal time impulsive control of sequential batch reactors with one or more species, SIAM J. on Control Optimization, 47, (6), (2008), 2827-2856.

[12] P.R. Wolenski And S. Zabić: A differential solution concept for impulsive systems, Dyn. Contin. Discrete \& Impuls. Syst, Supplementary volume DCDIS Series A: Application and Algorithms, ISSN 1201-3390. Proceedings 2 (The International Workshop on Differential Equations and Dynamical systems, Guelph, Canada july 29-31, 2006), (2006), 199-210.

[13] P.R. Wolenski AND S. Zabić: A sampling method and approximation results for impulsive systems, SIAM J. Control Optim., 46, (3), (2007), 983-998.

[14] Pontryagin L.S., V.G. Boltyanskit, R.V. Gamkrelidze, and E.F. Mishchenko: The mathematical theory of optimal processes, Translated from the Russian by K. N. Trirogoff; edited by L. W. Neustadt. Interscience Publishers John Wiley \& Sons, Inc. , New York-London, (1962). 
[15] Smith H.L. And P. Waltman: The Theory of the Chemostat, Cambridge University Press, (1995). 